Pesq. Vet. Bras. 35(6):518-524, junho 2015

DOI: $10.1590 / \mathrm{S} 0100-736 \mathrm{X} 2015000600005$

\title{
Celularidade do leite e Unidades Formadoras de Colônias nas mastites causadas por Staphylococcus coagulase positiva e coagulase negativa ${ }^{1}$
}

\author{
Helio Langoni ${ }^{2 *}$, Felipe Freitas Guimarães ${ }^{2}$, Elizabeth Oliveira da Costa ${ }^{3}$, \\ Samea Fernandes Joaquim ${ }^{2}$ e Benedito Donizete Menozzi ${ }^{2}$
}

\begin{abstract}
Langoni H., Guimarães F.F., Costa E.O., Joaquim S.F. \& Menozzi B.D. 2015. [Milk cellularity and colony forming units in mastitis caused by coagulase-positive staphylococci and coagulase negative.] Celularidade do leite e Unidades Formadoras de Colônias nas mastites causadas por Staphylococcus coagulase positiva e coagulase negativa. Pesquisa Veterinária Brasileira 35(6):518-524. Departamento de Higiene Veterinária e Saúde Pública, Faculdade de Medicina Veterinária e Zootecnia, Universidade Estadual Paulista, Distrito de Rubião Junior s/n, Botucatu, SP 18618-970, Brazil. E-mail: hlangoni@fmvz.unesp.br

Mastitis is the main affection of cattle intended for dairy production, which significantly impacts the milk production chain, with consequences yet to public health. It was studied aspects related to etiology, cellularity and bacterial count in 10 dairy farms, in the State of São Paulo. There were examined 1148 milking cows, totaling 4584 mammary glands. Cases in which there was isolation coagulase positive staphylococci (CPS) and coagulase-negative staphylococci (CNS) were considered. The results showed microbiota with various different pathogens and CNS (128 cases) and CPS (45), Staphylococcus aureus (90), Streptococcus agalactiae (70), Streptococcus dysgalactiae (69), Streptococcus uberis (29), Corynebacterium spp . (230), Klebsiella pneumoniae (28), Klebsiella oxytoca (2), Escherichia coli (15), Enterobacter spp. (3). The somatic cell count (SCC) related to CPS and CNS showed no differences among the evaluated properties, although with significant differences when evaluating the SCC between both groups of staphylococci, as evidenced by comparing discreet CNS and exuberant CPS (P $<0.01)$, discreet CPS and exuberant CPS $(\mathrm{P}<0.001)$ and moderate SCN and exuberant SCP (P $<0.01$ ). The evaluation of SCC was related to the intensity of infection, considering how slight growth isolation of up to nine colonies, moderate ten to 29 colonies and lush, with 30 or more colonies, revealed for both groups of staphylococci that the higher the number of colony forming units (CFU), SCC is higher being larger in cases of CPS. The results indicate that the importance of both CPS and CNS when considering the high occurrence of the evaluated properties and the fact that it raises the SCC, which compromises the quality of milk. We conclude that when there is a larger number of CFU, is concomitantly higher $\mathrm{SCC} / \mathrm{ml}$ of milk, in the case of CPS and CNS, which shows a direct relationship of the intensity of the infectious process with the response of milk cellularity, as well as the relevance of these in the etiology of mastitis and negatives aspects to both the production and the quality of milk produced in properties.
\end{abstract}

INDEX TERMS: Milk quality, staphylococci, cellularity, colony forming units.

${ }^{1}$ Recebido em 8 de agosto de 2014.

Aceito para publicação em 8 de junho 2015.

${ }^{2}$ Departamento de Higiene Veterinária e Saúde Pública, Faculdade de Medicina Veterinária e Zootecnia (FMVZ), Universidade Estadual Paulista (Unesp), Campus de Botucatu, Distrito de Rubião Junior s/n, Botucatu, SP 18618-970, Brasil. *Autor para correspondência: hlangoni@fmvz.unesp.br

${ }^{3}$ Departamento de Medicina Veterinária Preventiva e Saúde Animal, Faculdade de Medicina Veterinária e Zootecnia (FMVZ), Universidade de São Paulo (USP), Av. Prof. Dr. Orlando Marques de Paiva 87, Cidade Universitária, São Paulo, SP 05508-000, Brasil.
RESUMO.- A mastite é a principal afecção do gado destinado à produção leiteira, que impacta significativamente a cadeia produtiva do leite, com reflexos ainda para a saúde pública. Estudou-se aspectos relacionados à etiologia, celularidade e de contagem bacteriana em 10 propriedades leiteiras, localizadas no Estado de São Paulo. Foram examinadas 1148 vacas em lactação, totalizando 4584 glândulas mamárias. Foram considerados os casos, em que houve isolamento de estafilococos coagulase positiva (SCP) e estafi- 
lococos coagulase negativa (SCN). Os resultados revelaram microbiota com vários patógenos e diferentes espécies de SCN (128 casos) e SCP (45), Staphylococcus aureus (90), Streptococcus agalactiae (70), Streptococcus dysgalactiae (69), Streptococcus uberis (29), Corynebacterium spp. (230), Klebsiella pneumoniae (28), Klebsiella oxytoca (2), Escherichia coli (15), Enterobacter sp. (3). Os resultados de contagem de células somáticas (CCS) relacionados aos SCP e SCN não mostraram diferenças entre as propriedades avaliadas, entretanto com diferenças significantes ao se avaliar a CCS entre os dois grupos de estafilococos, como pode ser evidenciado ao comparar SCN Discreto e SCP exuberante $(\mathrm{P}<0,01)$, SCP Discreto e SCP exuberante $(\mathrm{P}<0,001)$ e SCN moderado e SCP exuberante $(\mathrm{P}<0,01)$. A avaliação da CCS relacionada à intensidade da infecção, considerando-se como crescimento discreto o isolamento de até nove colônias, moderado de dez a 29 colônias e exuberante, com 30 ou mais colônias, revelou para ambos os grupos de estafilococos que quanto maior o número de unidades formadoras de colônias (UFC), a CCS é mais elevada, sendo sempre maior nos casos de SCP. Conclui-se que quando há maior número de UFC, há concomitantemente maior CCS/ $\mathrm{mL}$ de leite, no caso dos SCP e SCN, o que mostra relação direta da intensidade do processo infeccioso com a resposta da celularidade do leite, bem como pela relevância desses na etiologia das mastites e dos aspectos negativos tanto para a produção, quanto na qualidade do leite produzido nas propriedades.

TERMOS DE INDEXAÇÃO: Qualidade do leite, estafilococos, celularidade, unidades formadoras de colônias.

\section{INTRODUÇÃO}

A mastite é reconhecida como a principal doença nos rebanhos leiteiros, pelas perdas econômicas tanto para o produtor como indústria de laticínios, decorrentes tanto da diminuição da produção leiteira como da qualidade do leite (Langoni 2013). Aliado aos aspectos de produtividade ressalta-se, ainda, os relacionados à saúde pública (Sá et al. 2004, De Freitas Guimarães et al. 2013).

De ampla etiologia microbiana (Langoni et al. 1998, Pitkälä et al. 2004) tem origem contagiosa e ambiental. As contagiosas são as causadas por micro-organismos conhecidos como "vaca dependentes" que tem como reservatório a glândula mamária como Staphylococcus aureus, várias espécies de Staphylococcus coagulase negativa, Streptococcus agalactiae, Corybacterium bovis e Mycoplasma spp. (Fox et al. 2003, Langoni 2013, Gonçalves et al. 2014, Tomazi et al. 2014), que são transmitidas a partir de vacas infectadas para outras no momento da ordenha pelas mãos dos ordenadores, equipamentos de ordenha e outros fômites de uso comum entre os animais, e as ambientais, que são causadas por micro-organismos, cuja fonte primária é o próprio ambiente onde os animais são mantidos, como camas, sala de ordenha, entre outros, e a transmissão ocorre principalmente após a ordenha. Nesse caso, tem-se as enterobactérias, como Escherichia coli, Klebsiella pneumoniae, Enterobacter spp, Streptococcus uberis e Streptococcus dysgalactiae, Pseudomonas spp.,
Trueperella pyogenes e as espécies leveduras, fungos e algas, como as do gênero Prototheca (Contreras \& Rodríguez 2011).

Uma outra classificação refere-se à gravidade do processo infeccioso e os micro-organismos são classificados em patógenos principais ou primários e os secundários, ou ainda, outros pesquisadores referem-se a patógenos maiores ou menores. Os principais são os mais virulentos com importância maior tanto para a saúde do animal como na qualidade do leite (Botaro et al. 2015, Beuron et al., 2014). Os secundários são considerados como parte da microbiota do úbere e tidos como de menor virulência, com menor efeito nos parâmetros que podem monitorar a qualidade do leite como a CCS. Entre os patógenos secundários estão, por exemplo, os Staphylococcus coagulase negativa (SCN) e admite-se atualmente a sua importância nas mastites relacionada tanto à diminuição de produção (Tenhagen et al. 2006) como produção de enterotoxinas e resistência a antimicrobianos (De Freitas Guimarães et al. 2013, Silva et al. 2014).

Entre os testes diagnósticos das mastites, a CCS, pode ser considerada como um marcador importante de processos inflamatórios e indicada para o monitoramento da qualidade do leite e saúde da glândula mamária. Além de possibilitar a detecção de mastite subclínica em nível de rebanho, prevê estimativas de perdas de produção láctea devido às mastites. Thiers et al. (2001) salientam que a CCS reflete a extensão do processo inflamatório e que a sua contagem diferencial, caracterizando os neutrófilos polimorfonucleares, os mononucleares e as células epiteliais caracterizam o estágio da inflamação, ou seja, a evolução do processo inflamatório. Os resultados de pesquisa referente à CCS mostram que além do seu aumento nos casos de mastite, também há alterações na composição do leite (dos Reis et al. 2013). 0 California Mastitis Test (CMT) relaciona-se também com à CCS e é considerado como teste indireto para o seu diagnóstico (Schalm \& Noorlander 1957).

0 número de unidades formadoras de colônias (UFC) é outro parâmetro importante para se avaliar a qualidade do leite produzido em propriedades leiteiras, que pode estar relacionado à ocorrência de mastites e também pela contaminação durante o processo de obtenção do leite, ou seja, os cuidados higiênicos estabelecidos no processo de ordenha, seja a nível animal, ordenhadeira e ambiental (Langoni et al. 2011, Botaro et al. 2013). Os procedimentos higiênicos na cadeia produtiva do leite são pontos críticos para a obtenção de matéria prima de qualidade.

Considerando a importância dos Staphylococcus coagulase negativa (SCN) e Staphylococcus coagulase positiva (SCP) como patógenos nas mastites, avaliou-se a CCS e UFC em amostras de leite de vacas com mastites por esses micro-organismos.

\section{MATERIAL E MÉTODOS}

Propriedades rurais e animais. Foram utilizadas 10 propriedades rurais, escolhidas por conveniência e interesse de seus proprietários, localizadas no Estado de São Paulo, todas com sistema de ordenha mecânica e com número variável de 100 a 300 vacas em lactação, todas da raça holandesa variedade preto e 
branco. Foram examinadas 1148 vacas em lactação, totalizando 4584 glândulas mamárias. Individualmente, realizaram-se testes de triagem (Tamis e CMT) totalizando 1318 amostras de leite de animais com mastite.

Diagnóstico do processo inflamatório e coleta de amostras. Foi realizado o teste do CMT de acordo com Schalm \& Noorlander (1957), colhendo-se amostras com escores de uma a três cruzes após lavagem do teto com água corrente e secagem com papel toalha descartável e desinfecção do óstio do teto com álcool iodado $(2,5 \%)$ em frascos esterilizados e transportados em caixas isotérmicas contendo gelo reciclável. Foram colhidas, ainda, amostras individuais de ao redor de $25 \mathrm{~mL}$ de leite de todas as glândulas mamárias positivas ao CMT para CCS em frascos contendo conservante celular Bronopol (Bertrand 1996). A CCS foi realizada por citometria de fluxo em equipamento Bentley (1995), Somacount $300^{\circledR}$.

Cultivo microbiológico. Foram utilizados os meios ágar-sangue ovino a 5\% e ágar McConkey, dividindo a placa em quatro quadrantes, inoculando $0,01 \mathrm{~mL}$ de cada amostra de leite, incubando em condições de aerobiose com leituras às 24, 48 e 72 horas de incubação, a $37^{\circ} \mathrm{C}$. Para avaliação das características fenotípicas dos isolados foi realizado estudo bacterioscópico pelos métodos de Gram e repique para caldo-cérebro-coração (BHI) para estudo bioquímico com caracterização das espécies dos isolados. No caso de suspeita de SCN e SCP, procedeu-se a contagem das UFC, categorizando-se em crescimento discreto (isolamento de até 9 colônias), moderado (10 a 29 colônias) e exuberante (30 ou mais colônias).

Identificação de Staphylococcus coagulase negativa (SCN) e Staphylococcus coagulase positiva (SCP) Após a análise morfo-tintorial, cocos Gram positivos foram submetidos a provas de catalase e coagulase (Koneman et al. 2008). 0 gênero Staphylococcus foi diferenciado de Micrococcus, com base nas provas de oxidação e fermentação da glicose, e pela resistência à bacitracina $(0,04 \mathrm{U})$, indicada pela ausência de halo de inibição ou formação de halo de até $9 \mathrm{~mm}$ e pela sensibilidade à furazolidona (100mg), caracterizada por halos de inibição de 15 a $35 \mathrm{~mm}$ de diâmetro (Baker 1984).

Para a identificação das espécies de SCN foi utilizado o método de Kloos \& Schleifer (1975), modificado por Cunha et al. (2004), que consiste na fermentação dos açúcares xilose, sacarose, trealose, maltose e manitol, produção de hemolisina e multiplicação em condições de anaerobiose em caldo tioglicolato. As linhagens foram mantidas a $37^{\circ} \mathrm{C}$ por 72 horas. Quando necessário e de acordo com os resultados obtidos pelo método modificado por Cunha et al. (2004), para complementar a classificação das demais espécies e subespécies de SCN também foram utilizadas outras provas bioquímicas, como a redução de nitrato, produção de urease e/ou ornitina descarboxilase, fermentação de $\beta$-D-frutose e resistência à novobiocina. Enquanto que, os isolados SCP foram submetidos a provas bioquímicas de fermentação dos açúcares trealose, manitol, maltose, pela resistência à polimixina B (300 UI) e produção de acetoína (Quinn et al., 2005). Posteriormente à identificação da espécie, os isolados foram conservados em caldo cérebro-coração com glicerol $\left(-80^{\circ} \mathrm{C}\right)$.

Cepas de referência internacional de espécies de SCN foram utilizadas como controle: Staphylococcus epidermidis (ATCC12228), S. simulans (ATCC 27851), S. warneri (ATCC 10209), S. xylosus (ATCC 29979) e S. saprophyticus (ATCC 15305).

Análise estatística. As análises estatísticas foram realizadas com Graphpad Instat software (Statistical Analysis Systems for Personal Computers, 2003), utilizando os testes de Kruskal-Wallis -ANOVA não paramétrica, teste de Mann-Whitney e teste exato de Fisher.

\section{RESULTADOS}

\section{Micro-organismos isolados}

Apesar do escopo do presente estudo estar relacionado ao gênero Staphylococcus spp. foram encontrados outros patógenos causadores de mastite, tanto contagiosos como ambientais, que estão apresentados no Quadro 1, com respectivas porcentagens de ocorrência em relação ao total das amostras microbiologicamente positivas e em relação ao gênero e espécie.

\section{Espécies de Staphylococcus coagulase negativa (SCN) e Staphylococcus coagulase positiva (SCP)}

A distribuição de resultados de identificação das espécies dos 128 SCN, de acordo com as propriedades avaliadas encontra-se no Quadro 2. Pode-se verificar maior frequência de mastites por SCN nas fazendas II $(27,3 \%)$ e III $(26,7 \%)$ e as mais baixas nas fazendas X $(0,8 \%)$ e VIII $(1,6 \%)$. A diferença na frequência de isolamento de SCN nas fazendas II e III foi estatisticamente significante quando comparada com a das fazendas VIII e X.

Quanto aos resultados referentes às espécies de SCP, é possível observar maior frequência de casos de mastites por SCP nas fazendas I $(13,6 \%)$ e fazenda X $(44,4 \%)$ e as mais baixas nas fazendas VI (1,5\%) e VII $(0,7 \%)$. As diferenças entre estas foram estatisticamente significantes, de acordo com o Quadro 3. A ocorrência de Staphylococcus aureus na fazenda X foi estatisticamente superior ao observado nas outras fazendas. Quando se analisou a ocorrência total nas dez fazendas o isolamento de $S$. aureus foi superior a $S$. intermedius, $S$. hyicus e $S$. schleiferi subsp coagulans e as diferenças

\section{Quadro 1. Frequência dos principais agentes etiológicos de mastite bovina em propriedades leiteiras do estado de} São Paulo. Botucatu, 2014

\begin{tabular}{lccc}
\hline $\begin{array}{l}\text { Microbiológico } \\
\text { quartos mamários }\end{array}$ & No & $\begin{array}{c}\text { Espécies em } \\
\text { relação ao } \\
\text { gênero }\end{array}$ & $\begin{array}{c}\text { Relacionado } \\
\text { ao total } \\
\text { microbiológico }\end{array}$ \\
\hline S. aureus & 90 & 34,2 & $\%$ \\
SCP* & 45 & 17,1 & 12,5 \\
SCN** & 128 & 48,7 & 6,2 \\
Staphylococcus total & 263 & & 17,7 \\
Streptococcus agalactiae & 70 & 41,7 & 36,4 \\
Streptococcus dysgalactiae & 69 & 41,1 & 9,7 \\
Streptococcus uberis & 29 & 17,3 & 9,5 \\
Streptococcus spp. & 168 & & 4,0 \\
Corynebacterium spp. & 230 & & 23,3 \\
Klebsiella pneumonia & 28 & 58,3 & 31,8 \\
Klebsiella oxytoca & 2 & 4,2 & 3,9 \\
Escherichia coli & 15 & 31,3 & 0,3 \\
Enterobacter sp. & 3 & 6,3 & 2,1 \\
Enterobacteriaceae & 48 & & 0,4 \\
Outros micro-organismos*** & 13 & & 6,7 \\
Total microbiológico positivo & 722 & & 1,8 \\
\hline
\end{tabular}

(Amostras contaminadas: Uma na Faz.I, três na Faz.II, uma na Faz.VI, três na Faz.VII). *SCP (estafilococos coagulase positiva): Staphylococus intermedius 21 (8\%), S. hyicus 19 (7,2\%), S. schleiferi subsp. coagulans $5(1,9$ $\%)$. ** SCN (estfilococos coagulase negativa) espécies identificadas no Quadro 6. *** Faz.III: Candida spp. (1), Pasteurella spp. (2), Nocardia spp. (1), Citrobacter spp. (1); Faz.VI: Candida spp. (1), Pasteurella spp. (3); Faz.VII: Nocardia spp. (1), Pseudomonas aeruginosa (1); Faz.VIII: Candida spp. (1), Citrobacter spp. (1). 
Quadro 2. Espécies de SCN de amostras de leite de casos de mastite bovina em dez propriedades do estado de São Paulo. Botucatu, 2014

\begin{tabular}{|c|c|c|c|c|c|c|c|c|c|c|c|c|}
\hline \multirow{2}{*}{$\begin{array}{l}\text { SCN } \\
\text { Espécies }\end{array}$} & \multicolumn{10}{|c|}{ Fazendas } & \multirow[t]{2}{*}{ Total } & \multirow[t]{2}{*}{$\%$} \\
\hline & $\mathrm{I}$ & II & III & IV & $\mathrm{V}$ & $\mathrm{VI}$ & VII & VIII & IX & $\mathrm{X}$ & & \\
\hline S. auriculares & 0 & 1 & 0 & 0 & 0 & 0 & 0 & 0 & 0 & 0 & 1 & 0,8 \\
\hline S. capitis & 0 & 1 & 0 & 0 & 0 & 0 & 0 & 0 & 0 & 0 & 1 & 0,8 \\
\hline S. chromogenes & 0 & 0 & 0 & 0 & 0 & 0 & 0 & 1 & 0 & 0 & 1 & 0,8 \\
\hline S. cohnii subsp. Cohnii & 0 & 2 & 1 & 0 & 1 & 0 & 0 & 0 & 0 & 0 & 4 & 3,1 \\
\hline S. epidermidis & 4 & 5 & 4 & 1 & 2 & 0 & 1 & 1 & 0 & 1 & 19 & 14,8 \\
\hline S. haemolyticus & 1 & 2 & 0 & 0 & 0 & 2 & 1 & 0 & 0 & 0 & 6 & 4,7 \\
\hline S. hominis & 0 & 0 & 2 & 3 & 0 & 0 & 0 & 0 & 1 & 0 & 6 & 4,7 \\
\hline S. hyicus & 2 & 7 & 3 & 2 & 1 & 0 & 0 & 0 & 1 & 0 & 16 & 12,5 \\
\hline S. lugdunensis & 0 & 0 & 1 & 0 & 0 & 1 & 0 & 0 & 0 & 0 & 2 & 1,6 \\
\hline S. pasteuri & 0 & 0 & 1 & 1 & 0 & 2 & 0 & 0 & 1 & 0 & 5 & 3,9 \\
\hline S. saccharolyticus & 1 & 0 & 1 & 0 & 0 & 0 & 0 & 0 & 0 & 0 & 2 & 1,6 \\
\hline S. saprophyticus subsp. Bovis & 0 & 0 & 0 & 0 & 0 & 1 & 0 & 0 & 0 & 0 & 1 & 0,8 \\
\hline S. saprophyticus subsp. Saprophyticus & 3 & 0 & 0 & 0 & 0 & 0 & 0 & 0 & 0 & 0 & 3 & 2,3 \\
\hline S. schleiferi subsp. Schleiferi & 1 & 1 & 1 & 3 & 0 & 0 & 0 & 0 & 0 & 0 & 6 & 4,7 \\
\hline S. sciuri subsp. Sciuri & 0 & 1 & 1 & 0 & 0 & 1 & 0 & 0 & 0 & 0 & 3 & 2,3 \\
\hline S. simulans & 0 & 1 & 1 & 1 & 0 & 0 & 1 & 0 & 0 & 0 & 4 & 3,1 \\
\hline S. warneri & 2 & 12 & 14 & 6 & 4 & 1 & 1 & 0 & 0 & 0 & 40 & 31,3 \\
\hline S. xylosus & 0 & 2 & 2 & 2 & 0 & 1 & 1 & 0 & 0 & 0 & 8 & 6,3 \\
\hline Total & 14 & 35 & 32 & 19 & 8 & 9 & 5 & 2 & 3 & 1 & 128 & 100 \\
\hline$\% \mathrm{SCN}$ & 10,9 & 27,3 & 26,7 & 14,8 & 6,3 & 7 & 3,9 & 1,6 & 2,3 & 0,8 & & \\
\hline
\end{tabular}

$\mathrm{P}=0.0001$, significante Kruskal-Wallis Teste (ANOVA não paramétrica);

$\mathrm{P}<0.05$ diferença significante na frequência de isolamento de SCN faz.II quando comparado com a faz.VIII; $\mathrm{P}<0.01$ diferença significante na frequência de isolamento de SCN faz.II quando comparado com a faz.X; $\mathrm{P}<0.05$ diferença significante na frequência de isolamento de SCN faz.III quando comparado com a faz.VIII; $\mathrm{P}<0.01$ diferença significante na frequência de isolamento de SCN faz.III quando comparado com a faz.X.

Quadro 3. Isolamentos de espécies de SCP de amostras de leite de casos de mastite bovina em dez propriedades do estado de São Paulo. Botucatu, 2014

\begin{tabular}{|c|c|c|c|c|c|c|c|c|c|c|c|c|}
\hline \multirow{2}{*}{$\begin{array}{l}\text { SCP } \\
\text { Espécies }\end{array}$} & \multicolumn{10}{|c|}{ Fazendas } & \multirow[t]{2}{*}{ Total } & \multirow[t]{2}{*}{$\%$} \\
\hline & $\mathrm{I}$ & II & III & IV & V & VI & VII & VIII & IX & $\mathrm{X}$ & & \\
\hline S. aureus & 2 & 0 & 4 & 2 & 1 & 1 & 1 & 11 & 8 & 60 & $90^{\mathrm{a}, \mathrm{d}, \mathrm{c}}$ & 68,2 \\
\hline S. intermedius & 9 & 2 & 4 & 2 & 3 & 0 & 0 & 0 & 1 & 0 & $21^{\mathrm{a}}$ & 15,2 \\
\hline S. hyicus & 4 & 3 & 4 & 3 & 2 & 1 & 0 & 0 & 2 & 0 & $19^{\mathrm{b}}$ & 12,9 \\
\hline $\begin{array}{l}\text { S. schleiferi subsp } \\
\text { coagulans }\end{array}$ & 3 & 0 & 1 & 1 & 0 & 0 & 0 & 0 & 0 & 0 & $5^{c}$ & 3,8 \\
\hline Total & $18^{\mathrm{d}, \mathrm{e}}$ & $5^{\mathrm{d}, \mathrm{e}}$ & $13^{\mathrm{d}}$ & $8^{d}$ & $6^{\mathrm{d}, \mathrm{e}}$ & $2^{\mathrm{d}, \mathrm{e}}$ & $1^{\mathrm{d}, \mathrm{e}}$ & $11^{\mathrm{d}}$ & $11^{\mathrm{d}}$ & $60^{\mathrm{d}}$ & 135 & 100 \\
\hline$\%$ SCP & 13,3 & 3,7 & 9,6 & 5,9 & 4,4 & 1,5 & 0,7 & 8,1 & 8,1 & 44,4 & & \\
\hline
\end{tabular}

$\mathrm{P}<0,0001$ ocorrência de Staphylococcus aureus na faz.X foi estatisticamente superior ao observado nas outras fazendas (Teste de Exato de Fisher aproximação de Katz). ${ }^{a} \mathrm{P}<0,0001$ isolamento de $S$. aureus foi su-

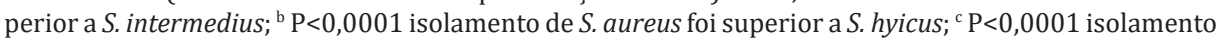
de $S$. aureus foi superior; ${ }^{\mathrm{d}} \mathrm{P}<0,0001$ frequência de isolamento na faz. $\mathrm{X}$ das espécies de SCP dos casos de mastite foi superior às demais fazendas; ${ }^{\mathrm{e}} \mathrm{P}<0,0001$ de isolamento na faz. I das espécies de SCP dos casos de mastite foi superior à fazenda VII; fazenda VI $(\mathrm{P}=0,0002)$ e faz.II $(\mathrm{P}=0,0076)$ e faz.V $(\mathrm{P}=0,0171)$.

foram estatisticamente significantes. Quando se comparou as frequências de isolamento das espécies de SCP dos casos de mastite verificou-se que a frequência de isolamento na fazenda $\mathrm{X}$ foram superiores às demais fazendas $(\mathrm{P}<0.0001)$, enquanto a frequência de isolamento de SCP na fazenda I foi estatisticamente superior às fazendas II, V, VI e VII.

\section{Contagem de células somáticas (CCS)}

Das 1318 amostras positivas na CCS, foram isolados Staphylococcus spp. em 263, sendo SCN $(n=128)$ e SCP ( $\mathrm{n}=135)$, de acordo com o Quadro 4, em que é possível avaliar a intensidade do quadro inflamatório.

Os resultados da intensidade da infecção avaliada pelo número de colônias (UFC) nos 128 isolados de SCN e 135 de SCP, relacionados à CCS, estão apresentados no Quadro 5 , em que considera-se crescimento discreto (até 9 colô- nias), moderado (10 a 29 colônias) e exuberante (30 colônias ou mais). É possível verificar que há diferença significante para SCN discreto e SCP exuberante $(\mathrm{P}<0,01)$, SCP discreto e SCP exuberante $(\mathrm{P}<0,001)$ e SCN moderado e SCP exuberante $(\mathrm{P}<0,01)$.

Quanto aos resultados de CCS relacionados às 14 espécies de SCN caracterizadas no presente estudo, de acordo com o Quadro 6, pode-se verificar que apesar de diferenças quanto a CCS entre elas, essa não é estatisticamente significativa $(\mathrm{p}=0,7204)$. Da mesma forma, os resultados de CCS relacionados às espécies de $\mathrm{SCP}$, também não revelou diferença significante $(\mathrm{p}=0,4617)$, de acordo com o Quadro 7.

\section{DISCUSSÃO}

A mastite continua sendo o principal entrave para a pecuária leiteira, considerando a elevada prevalência e os preju- 
ízos ocasionados à cadeia produtiva do leite. Os resultados obtidos mostram uma etiologia múltipla envolvendo diferentes grupos de micro-organismos (Langoni et al. 1998, Pitkälä et al. 2004). Especificamente o gênero que agrupa as espécies de Staphylococcus coagulase negativa (SCN) e Staphylococcus coagulase positiva (SCP), observou-se no presente estudo elevada ocorrência de micro-organismos

Quadro 4. Intensidade do processo inflamatório em relação à $\mathrm{CCS}$ em 263 casos de mastite bovina por micro-organismos do gênero Staphylococcus em 10 propriedades leiteiras do estado de São Paulo. Botucatu, 2014

\begin{tabular}{lcc}
\hline CCS & $\begin{array}{c}\text { SCN } \\
\text { (Coagulase negativo) } \\
\text { (células } \times 10^{3} / \mathrm{mL} \text { ) }\end{array}$ & $\begin{array}{c}\text { SCP } \\
\text { (Coagulase positivo) } \\
\text { (células x103/mL) }\end{array}$ \\
\hline Mediana & 424,5 & 792,0 \\
Desvio padrão & 1422,3 & 1584,2 \\
Erro padrão & 125,7 & 136,3 \\
Mínimo & 19,0 & 15,0 \\
Máximo & 8633,0 & 8650,0
\end{tabular}

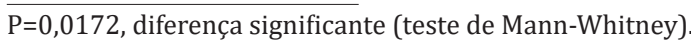

Quadro 5. Intensidade do processo inflamatório em CCS em relação à intensidade da infecção quanto ao número de colônias (UFC) de SCN e SCP isolados de dez propriedades leiteiras do estado de São Paulo. Botucatu, 2014

\begin{tabular}{lccc}
\hline $\begin{array}{l}\text { Número estimado } \\
\text { colônias (UFC) }\end{array}$ & $\begin{array}{c}\text { Mediana } \\
\text { CCS. } 10^{3} / \mathrm{mL}\end{array}$ & $\begin{array}{c}\text { Mínimo } \\
\text { CCS. } 10^{3} / \mathrm{mL}\end{array}$ & $\begin{array}{c}\text { Máximo } \\
\text { CCS. } 10^{3} / \mathrm{mL}\end{array}$ \\
\hline SCN Discreto (0-9 col.) & $342,00^{\mathrm{a}}$ & 19.000 & 5904.0 \\
SCP Discreto (0-9 col.) & $348,50^{\mathrm{b}}$ & 15.000 & 3322.0 \\
SCN Moderado (10-29 col.) & $356,50^{\mathrm{c}}$ & 45.000 & 3365.0 \\
SCP Moderado (10-29 col.) & 962,00 & 47.000 & 7496.0 \\
SCN Exuberante ( $\geq 30$ col.) & 560,50 & 50.000 & 8633.0 \\
SCP Exuberante ( $\geq 30$ col.) & $1187,5^{\text {abc }}$ & 46.000 & 8650.0
\end{tabular}

*Número estimado classificado como: exuberante ( $\geq$ de 30 ufc $/ 0,001 \mathrm{~mL}$ ); moderado (10-29 ufc/0,001mL); $\operatorname{discreto}(<10 \mathrm{cfc} / 0,001 \mathrm{ufc} / \mathrm{mL})$. $\mathrm{P}<0,0001$, significante Kruskal-Wallis Teste-ANOVA não paramétrica); ${ }^{a}$ $\mathrm{P}<0,01$ diferença significante SCN Discreto e SCP exuberante; ${ }^{\mathrm{b}} \mathrm{P}<0,001$ diferença significante entre SCP Discreto e SCP exuberante; ${ }^{c} \mathrm{P}<0,01$ diferença significante entre SCN moderado e SCP exuberante.

Quadro 6. Contagem de células somáticas de amostras de leite de glândulas mamárias bovinas infectadas por espécies de SCN das dez propriedades leiteiras de diferentes de regiões do estado de São Paulo. Botucatu, 2014

\begin{tabular}{lccc}
\hline SCN & $\begin{array}{c}\text { Mediana } \\
\text { CCS. } 10^{3} / \mathrm{mL}\end{array}$ & $\begin{array}{c}\text { Mínimo } \\
\text { CCS. } 10^{3} / \mathrm{mL}\end{array}$ & $\begin{array}{c}\text { Máximo } \\
\text { CCS. } 10^{3} / \mathrm{mL}\end{array}$ \\
\hline S. epidermidis & 465,0 & 19,0 & 2158,0 \\
S. hyicus & 472.0 & 48,0 & 8633,0 \\
S. warneri & 472,0 & 45,0 & 5904,0 \\
S. xylosus & 838,0 & 105,0 & 4465,0 \\
S. haemolyticus & 348,5 & 200,0 & 2308,0 \\
S. cohnii subsp cohnii & 436,0 & 387,0 & 1582,0 \\
S. pasteuri & 108,0 & 30,0 & 343,0 \\
S. sciuri & 1240,5 & 73,0 & 1373,0 \\
S. hominis & 459,0 & 22,0 & 6706,0 \\
S. schleiferi subsp schleiferi & 134,0 & 45,0 & 5324,0 \\
S. saprophyticus subsp sapro & 460,0 & 368,0 & 1000,0 \\
S. simulans & 712,0 & 310,0 & 1572,0 \\
S. saccharolyticus & 458,0 & 300,0 & 616,0 \\
S. lugdunensis & 207,5 & 86,0 & 329,0 \\
\hline
\end{tabular}

$\overline{\mathrm{P}=0,7204 \text { considerado não significante }}$ (Teste Kruskal-Wallis /ANOVA não paramétrico).
Quadro 7. Contagem de células somáticas de amostras de leite de glândulas mamárias bovinas infectadas por espécies de SCP das dez propriedades leiteiras de diferentes de regiões do estado de São Paulo. Botucatu, 2014

\begin{tabular}{lccc}
\hline SCP & $\begin{array}{c}\text { Mediana } \\
\text { CCS. } 10^{3} / \mathrm{mL}\end{array}$ & $\begin{array}{c}\text { Mínimo } \\
\text { CCS. } 10^{3} / \mathrm{mL}\end{array}$ & $\begin{array}{c}\text { Máximo } \\
\text { CCS. } 10^{3} / \mathrm{mL}\end{array}$ \\
\hline S. aureus & 972,5 & 26,0 & 8650,0 \\
S. intermedius & 1211,5 & 33,0 & 7496,0 \\
S.hyicus & 582,0 & 60,0 & 3322,0 \\
S. schleiferi subsp coagulans & 2199,0 & 15,0 & 5697,0
\end{tabular}

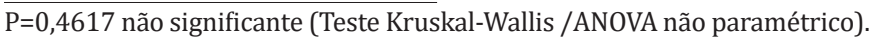

do gênero Staphylococcus (36,49\%), o que está de acordo com Costa et al. (2000) e da Cunha et al. (2004). Entre os SCP, Staphylococcus aureus foi encontrado em $34,2 \%$ entre as 10 propriedades avaliadas no presente estudo e é considerado como importante patógeno nas mastites por expressar grande variedade de fatores de virulência (Nader Filho et al. 2007, Piepers et al. 2007, Silva et al. 2013).

A elevada ocorrência de SCN (48,7\%) encontrada é também assinalada por outros pesquisadores como Piepers et al. (2007) e Tomazi et al. (2014) que encontraram SCN em $41 \%$ dos casos. Notadamente esses patógenos tem sido observados nas mastites subclínicas e clínicas com acentuada redução da produção leiteira (De Vliegher et al. 2005, Taponen et al. 2006, De Freitas Guimarães et al. 2013). De maneira geral, há uma variação percentual entre as diferentes espécies de SCN isoladas em diferentes estudos, entretanto, é evidente a importância desses como agentes infecciosos nas mastites (Brabes et al. 1999, Costa et al. 2008, De Freitas Guimarães et al. 2013, Silva et al. 2014).

Após a invasão da glândula mamária, os micro-organismos se multiplicam, lesando o tecido glandular com consequente resposta celular com elevação da CCS, seja de polimorfonucleares, monócitos e de células epiteliais, sendo de $80-90 \%$ a resposta por neutrófilos. A pesquisa de Benites et al. (2000) mostrou que nos processos inflamatórios agudos há uma resposta de $98,7 \%$ de polimorfonucleares e que 14,3\% de monócitos encontravam-se no lúmen alveolar e parênquima glandular e que por outro lado nos processos crônicos $51,9 \%$ eram mononucleares e apenas $48,4 \%$ polimorfonucleares.

Quando se comparou a CCS do leite nos casos de mastites causadas por SCP e SCN, encontrou-se maior valor no caso dos SCP (1 325,3 x $10^{3}$ células $\left./ \mathrm{mL}\right)$, significativamente superior à observada nos casos de SCN $\left(991,58 \times 10^{3}\right.$ células/mL). No Quadro 4, entretanto, pode-se observar que os valores máximos de CCS para ambos os grupos foram semelhantes, o que demonstra que os SCN provocam intensa resposta inflamatória ao avaliar a CCS (Philipsson et al. 1995, dos Reis et al. 2013, Tomazi et al. 2014).

A avaliação da CCS frente à intensidade do processo infeccioso caracterizado pelo número de colônias (UFC) tanto no caso das mastites por SCN e SCP revelou que quanto maior o número de UFC, a CCS é mais elevada, sendo sempre maior no caso dos SCP (Quadro 5). Detectaram-se diferenças significativas na comparação nos casos de SCP com crescimento discreto e exuberante $(\mathrm{P}<0,001)$. Espera-se que a carga bacteriana e os aspectos de patogenicidade e 
virulência exerçam efeito mais agressivo provocando uma maior resposta com relação às células de defesa, que na realidade representam principalmente os polimorfonucleares. 0 estudo de Costa et al. (2008) mostrou resposta celular significativa nos casos de mastite subclínica causadas por SCN, da mesma forma que nos casos clínicos de mastite.

Dentre as 14 espécies de SCN caracterizadas (Quadro 6) há uma variação com relação aos valores medianos de CCS e várias espécies apresentaram valores maiores e/ ou próximos a $10^{6}$ células $/ \mathrm{mL}$ de leite, como Staphylococcus hyicus, Staphylococcus xylosus, Staphylococcus hominis, Staphylococcus schleiferi subesp. schleiferi, Staphylococcus warneri, Staphylococcus sciuri subesp. sciuri. No caso de S. hominis detectou-se CCS superior a $2 \times 10^{6}$ células $/ \mathrm{mL}$ de leite e somente nos casos de Staphylococcus pasteuri e Staphylococcus lugdunensis a CCS foi inferior a 400000 células/mL de leite, considerado limite para qualidade do leite em alguns países. Da mesma forma, Costa et al. (2008) ressaltaram o papel dos SCN nas mastites, considerando os aumentos na CCS principalmente para Staphylococcus saprophyticus e $S$. warneri.

A CCS é um dos parâmetros internacionais de qualidade do leite, pois a celularidade do leite reflete diretamente a ocorrência de mastites no rebanho, impactando negativamente a produção, seja pela redução na produção de leite, como também, pela redução nos constituintes nutricionais, além dos prejuízos para a indústria, prejudicando a qualidade dos derivados lácteos, pois há um aumento da proteólise no leite com altas contagens de células somáticas, o que compromete a produção de derivados lácteos, principalmente de queijos (Seegers et al. 2003).

Os SCP e SCN são considerados como contagiosos e ao se avaliar a variação dos isolamentos nas propriedades estudadas pode-se discutir os aspectos relacionados, principalmente, ao manejo de ordenha com utilização de pré-dipping, assim como, pós ordenha com a aplicação de pós-dipping, de forma rotineira, além de higiene do ordenhador, incluindo a utilização de luvas durante a ordenha (Medeiros et al. 2009, Tomazi et al. 2014). Essas práticas possibilitam a diminuição da ocorrência de mastites por patógenos contagiosos. Outro aspecto é que algumas propriedades adotam programa de descarte de vacas positivas e repetidoras de mastite por $S$. aureus, o que diminui as chances de transmissão durante a ordenha. Sabe-se que o manejo da ordenha é variável entre as propriedades leiteiras havendo necessidade de adequações de acordo com as deficiências avaliadas (Langoni 2013).

\section{CONCLUSÕES}

É possível concluir pela importância tanto dos SCP como dos SCN como agentes de mastites, considerando-se a elevada frequência desses patógenos nos rebanhos pesquisados e pelo fato de determinar elevação na CCS, comprometendo a qualidade do leite oferecido para consumo.

A contagem de unidades formadoras de colônias nas infecções por SCP e SCN mostra relação direta do processo infeccioso com à celularidade representada pela CCS, pois para os dois grupos de micro-organismos verifica-se aumento na CCS de acordo com número de colônias (UFC) estabelecido no presente estudo.
Agradecimentos.- À FAPESP pela Bolsa de Mestrado de Felipe de Freitas Guimarães (Processo Fapesp 2008/08780-7) e auxílio à pesquisa (Processo Fapesp 2008/11614-1)

\section{REFERÊNCIAS}

Baker J.S. 1984. Comparison of various methods for differentiation of staphylococci and micrococci. J. Clin. Microbiol. 19:875-79.

Benites N.R., Melville P.A. \& Costa E.O. 2000. Feature and intensity of inflammatory responses in bovine mammary gland. Proc. Symposium on Immunolocy of Ruminant Mammary Gland Stress, Italy, p.30-35.

Bentley 1995. Somacount 300: operator's manual. Chasca.

Bertrand J.A. 1996. Influence of shipping container preservative and breed on analysis of milk components of shipped samples. J. Dairy Sci. 6(1):145-148

Beuron D.C., Cortinhas C.S., Botaro B.G., Macedo S.N., Gonçalves J.L., Brito M.A. \& Santos M.V. 2014. Risk factors associated with the antimicrobial resistance of Staphylococcus aureus isolated from bovine mastitis. Pesq. Vet. Bras. 34(10):947-952.

Brabes K.C.S., Carvalho E.P., Dionísio F.L., Pereira M.L., Garino Jr F. \& Costa E.O. 1999. Participação de espécies coagulase positivas e negativas produtoras de enterotoxinas do gênero Staphylococcus na etiologia de casos de mastite bovina em propriedades de produção leiteira dos Estados de São Paulo e Minas Gerais. Revta Napgama, São Paulo, 2:4-11.

Botaro B.G., Gameiro A.H. \& Santos M.V.D. 2013. Quality based payment program and milk quality in dairy cooperatives of Southern Brazil: an econometric analysis. Sci. Agric. 70(1):21-26.

Botaro B.G., Cortinhas C.S., Dibbern A.G., Silva L.F.P., Benites N.R. \& dos Santos M.V. 2015. Staphylococcus aureus intramammary infection affects milk yield and SCC of dairy cows. Trop. Anim. Health Prod. 47(1):61-66.

Contreras G.A. \& Rodríguez J.M. 2011. Mastitis: comparative etiology and epidemiology. J. Mammary Gland Biol. Neoplasia 16(4):339-356.

Costa E.O., Garino Jr F., Melville P.A., Ribeiro A.R., Silva J.A.B., Watanabe E.T. \& Valle C.R. 2000. Estudo da etiologia das mastites bovinas nas sete principais bacias leiteiras do Estado de São Paulo. Revta Napgama, São Paulo, 3(4):6-13.

Costa E.O., Guimarães F.F., Faccioli P.Y., Santos F., Langoni H., Arcaro J., Peres A. \& Cunha M.L. 2008. Identification the coagulase negative staphylococci isolates from bovine mastitis and evaluation of the inflammatory proccess. Oral and Poster Abstracts XXV Jubilee World Buiatrics Congress. Alapította, Budapest, 130:73-73.

Cunha M.L.R.S., Sinzato Y.K. \& Silveira L.V.A. 2004. Comparison of methods for the identification of coagulase-negative Staphylococci. Mem. Inst. Oswaldo Cruz 99(8):855-860.

De Freitas Guimarães F., Nóbrega D.B., Richini-Pereira V.B., Marson P.M., De Figueiredo Pantoja J.C. \& Langoni H. 2013. Enterotoxin genes in coagulase-negative and coagulase-positive staphylococci isolated from bovine milk. J. Dairy Sci. 96:2866-2872.

De Vliegher S., Barkema H.W., Opsomer G., De Kruif A. \& Duchateau L. 2005. Association between somatic cell count in early lactation and culling of dairy heifers using Cox frailty models. J. Dairy Sci. 88(2):560-568.

Dos Reis C.B.M., Barreiro J.R., Mestieri L., de Felício Porcionato M.A. \& dos Santos M.V. 2013. Effect of somatic cell count and mastitis pathogens on milk composition in Gyr cows. BMC Vet Res. 9(1):67.

Fox L.K., Hancock D.D., Mickelson A., Britten A. \& Kaaden O.R. 2003. Bulk tank milk analysis: factors associated with appearance of Mycoplasma sp. in milk. J. Vet. Med. B 50(5):235-240.

Gonçalves J.L., Tomazi T., Barreiro J.R., De Campos Braga P.A., Ferreira C.R., Araújo Junior J.P. \& Dos Santos M.V. 2014. Identification of Corynebacterium spp. isolated from bovine intramammary infections by matrix-assisted laser desorption ionization-time of flight mass spectrometry. Vet. Microbiol. 173(1):147-151.

Kloos W.E. \& Schleifer K.H. 1975. Isolation and characterization of staphylococci from human skin, descriptions of four new species: Staphylococcus warneri, Staphylococcus capitis, Staphylococcus hominis, Staphylococcus simulans. Int. J. Syst. Bacteriol. 25:62-79. 
Koneman E.W., Allen S.D., Janda W.M., Schreckenberger P.C. \& Winn Jr W.C. 2008. Diagnóstico Microbiológico: texto e atlas colorido. 6⿳亠丷a ed. Guanabara Koogan, Rio de Janeiro.

Langoni H. 2013. Qualidade do leite: utopia sem um programa sério de monitoramento da ocorrência de mastite bovina. Pesq. Vet. Bras. 33:620-626.

Langoni H., Da Silva A.V., Cabral K.G. \& Domingues P.F. 1998. Aspectos etiológicos na mastite bovina: flora bacteriana aeróbica. Revta Bras. Med. Vet. 20(5):204-210.

Langoni H., Penachio S., Citadella J.C.C., Laurino F., Faccioli-Martins P.Y., Lucheis S.B., Menozzi B.D. \& Silva A.V. 2011. Aspectos microbiológicos e de qualidade do leite bovino. Pesq. Vet. Bras. 31:1059-1065.

Medeiros E.S.D., Santos M.V.D., Pinheiro Júnior J.W., Faria E.B.D., Wanderley G.G., Teles J.A.A. \& Mota R.A. 2009. Avaliação in vitro da eficácia de desinfetantes comerciais utilizados no pré e pós-dipping frente amostras de Staphylococcus spp. isoladas de mastite bovina: in vitro evaluation of the efficacy of commercial disinfectants used in pre and post-dipping against Staphylococcus spp. isolated from bovine mastitis:[revision]. Pesq. Vet. Bras, 29(1):71-75.

Nader Filho A., Ferreira L.M., Do Amaral L.A., Junior O.R. \& Oliveira R.P. 2007. Sensibilidade antimicrobiana dos Staphylococcus aureus isolados no leite de vacas com mastite. Arqs Inst. Biológico, São Paulo, 74(1):1-4.

Philipsson J., Ral G. \& Berglund B. 1995. Somatic cell count as a selection criterion for mastitis resistance in dairy cattle. Livest. Prod. Sci. 41:195200.

Piepers S., De Meulemeester K., De Aart L., Opsomer G., Barkema H.W. \& Vliegher S. 2007. Prevalence and distribution of mastitis pathogens in subclinically infected dairy cows in Flanders, Belgium. J. Dairy Res. 74:478-483.

Pitkälä A., Haveri M., Pyörälä S., Myllys V. \& Honkanenbuzalski T. 2004. Bovine Mastitis in Finland 2001 - prevalence, distribution of bacteria, and antimicrobial resistance. J. Dairy Sci. 87:2433-2441.

Quinn P.J., Markey B., Carter M.E., Donnelly W.J. \& Leonard F.C. 2005. Microbiologia Veterinária e Doenças Infecciosas. Ed. Artmed, Porto Alegre.
Sá M.E.P., Cunha M.L.R.S., Elias A.O., Victoria C. \& Langoni H. 2004. Importância do Staphylococcus aureus nas mastites subclínicas: pesquisa de enterotoxinas e toxina do choque tóxico, e a relação com a contagem de células somáticas. Braz. J. Vet. Res. Anim. Sci. 41(5):320-326.

Schalm G.N. \& Noorlander D.D. 1957. Experiments and observations leading to development of the California Mastitis Test. J. Am. Med. Assoc. 130:199-204.

Seegers H., Fourichon C. \& Beaudeau F. 2003. Production effects related to mastitis and mastitis economics in dairy cattle herds. Vet. Res. 34:475491.

Silva N.C.C., Guimarães F.F., Manzi M.P., Budri P.E., Gómez-Sanz E., Benito D., Langoni H., Rall V.L.M. \& Torres C. 2013. Molecular characterization and clonal diversity of methicillin-susceptible Staphylococcus aureus in milk of cows with mastitis in Brazil. J. Dairy Sci. 96:6856-6862.

Silva N.C.C., Guimarães F.F., Manzi M.P., Gómez-Sanz E., Gómez P., AraújoJunior J.P., Langoni H., Rall V.L.M. \& Torres C. 2014. Characterization of methicillin-resistant coagulase-negative staphylococci in milk from cows with mastitis in Brazil. Antonie van Leeuwenhoek 106:227-233.

Statistical Analysis Systems for Personal Computers. 2003. Graphpad Instat software. 1990-1993.

Taponen S., Simojoki H., Haveri M., Larsen H.D. \& Pyorala S. 2006. Clinical characteristics and persistence of bovine mastitis caused by different species of coagulase-negative staphylococci identified with API or AFLP. Vet. Microbiol. 115:199-207.

Tenhagen B.A., Köster G., Wallmann J. \& Heuwieser W. 2006. Prevalence of mastitis pathogens and their resistance against antimicrobial agents in dairy cows in Brandenburg, Germany. J. Dairy Sci. 89:2542-2551.

Thiers F.O., Benites N.R. \& Costa E.O. 2001. Proporção de polimorfonucleares neutrófilos (PMN), mononucleares (MN) no leite comparados com os escores de CMT. Revta Napgama, São Paulo, 4(3):3-5.

Tomazi T., Gonçalves J.L., Barreiro J.R., De Campos Braga P.A., Silva L.F.P., Eberlin M.N. \& dos Santos M.V. 2014. Identification of coagulase-negative staphylococci from bovine intramammary infection by matrix-assisted laser desorption ionization-time of flight mass spectrometry. J. Clin. Microbial. 52(5):1658-1663. 